\title{
Properties of hyperholomorphic functions and integrals for commutative-quaternionic valued functions
}

\author{
Ji Eun Kim
}

Department of Mathematics, Dongguk University, Gyeongju, Republic of Korea.

\author{
Communicated by P. Kumam
}

\begin{abstract}
We give representations and properties of a hyperholomorphic function with values in commutative-quaternions. We first consider expressions of commutative-quaternions. Also, we investigate the results of derivatives and integrations for a hyperholomorphic function of commutative-quaternionic variables in Clifford analysis.
\end{abstract}

Keywords: Hyperholomorphic function, commutative-quaternions, Cauchy-Riemann system, Clifford analysis.

2010 MSC: 32A99, 32W50, 30G35, 11E88.

(C)2018 All rights reserved.

\section{Introduction}

A quaternion is a hypercomplex number defined in four dimensions which is introduced by Hamilton [4]. It can be represented as follows:

$$
z=x_{0}+x_{1} i+x_{2} j+x_{3} k, \quad x_{r} \in \mathbb{R}, \quad r=0,1,2,3,
$$

where $\mathbb{R}$ is a set of real numbers and the noncommutative units $i, j$, and $k$ satisfy the relations:

$$
\mathfrak{i}^{2}=j^{2}=k^{2}=-1, \quad i j=-j i=k, \quad j k=-k j=i, \quad k i=-i k=j .
$$

Unlike in the complex analysis, the two analogous ways of defining a holomorphic functions of a quaternionic variable which are not equivalent, as in [10]. Sudbery [14] developed and organized the analogous statements for quaternionic analysis. The algebra of quaternions $\mathbb{H}$ has developed the new theory which can be applied in physics. Because quaternions has the noncommutative properties of the multiplication, the Cauchy integral formula which is produced by Fueter [2] has two versions, left and right regular

Email address: jeunkim@pusan.ac.kr (Ji Eun Kim)

doi: $10.22436 /$ jnsa.011.04.03

Received: 2017-09-12 Revised: 2018-01-24 Accepted: 2018-01-25 
quaternionic functions for each analogue of the complex holomorphic functions satisfying the CauchyRiemann equations:

$$
\overline{\mathrm{D}} \mathrm{f}:=\frac{\partial f}{\partial x_{0}}+i \frac{\partial f}{\partial x_{1}}+j \frac{\partial f}{\partial x_{2}}+k \frac{\partial f}{\partial x_{3}}=0
$$

and

$$
f \bar{D}:=\frac{\partial f}{\partial x_{0}}+\frac{\partial f}{\partial x_{1}} i+\frac{\partial f}{\partial x_{2}} j+\frac{\partial f}{\partial x_{3}} k=0,
$$

where $f: \mathbb{H} \rightarrow \mathbb{H}$ is a differentiable function. Nôno [11] studied the properties of quaternions and the definition of hyperholomorphic functions of quaternion variables. Kajiwara et al. [5] gave a basic calculation for inhomogeneous Cauchy-Riemann system and the theories on a densely defined some operators in a Hilbert space. Kim and Shon [7] researched unusual equations corresponding Cauchy-Riemann systems and properties of functions with values in special quaternions by using a regular function with values in dual split quaternions. Kim et al. [8,9] obtained the regularity of functions on the reduced quaternion field, and for the regularities of functions on the form of dual split quaternions.

Hypercomplex systems whose multiplications are commutative, which are introduced by Kaledin [6], were applied by the development of the theory of functions of a complex variable. Scheicher et. al. [13] extended some known inequalities for complex numbers to certain systems of hypercomplex numbers. Fjelstad and Gal [1] generalized the complex analyticity to hypercomplex functions which included the standard complex definition, except for reducing analytic functions to a trivial class. Grant and Strachan [3] described complex numbers in the $n$ dimension by using hypercomplex bases and the variables in real numbers. Olariu [12] presented a detailed analysis of the hypercomplex numbers and the properties of polar and planar hypercomplex numbers in the $n$ dimension. Olariu researched the interplay between the algebraic, the geometric, and the analytic relations.

In this paper, we describe the basic notation and definitions of the commutative-quatnions in four dimensions, for which the multiplication is associative and commutative. Also, we investigate properties of a hyperholomorphic function and some representations of integral formulas for commutativequaternionic valued functions.

\section{Preliminaries}

A commutative-quaternion is determined as the ordered pair by four real components $\left(x_{0}, x_{1}, x_{2}, x_{3}\right)$. Let $i, j$, and $k$ denote the pure imaginary numbers, respectively, then it is represented by

$$
p=x_{0}+i x_{1}+j x_{2}+k x_{3}
$$

where $i, j$, and $k$ are bases for which the product rules are

$$
\mathfrak{i}^{2}=\mathfrak{j}^{2}=-1, \quad k^{2}=1, \quad \mathfrak{j}=j i=-k, \quad i k=k i=j, \quad j k=k j=i .
$$

Then we give a set of commutative-quaternions:

$$
B Q:=\left\{p=x_{0}+i x_{1}+j x_{2}+k x_{3} \mid x_{r} \in \mathbb{R}(r=0,1,2,3)\right\},
$$

which is isomorphic to two dimensional complex numbers. If $x_{0}=y_{0}, x_{1}=y_{1}, x_{2}=y_{2}$, and $x_{3}=y_{3}$, then two commutative-quaternions $p=x_{0}+i x_{1}+j x_{2}+k x_{3}$, and $q=y_{0}+i y_{1}+j y_{2}+k y_{3}$ are said to be equal. For $p, q \in B Q$, the sum $p+q$ and the product $p q$ can be expressed by

$$
p+q=\left(x_{0}+y_{0}\right)+i\left(x_{1}+y_{1}\right)+j\left(x_{2}+y_{2}\right)+k\left(x_{3}+y_{3}\right)
$$

and

$$
\begin{aligned}
p q= & \left(x_{0} y_{0}-x_{1} y_{1}-x_{2} y_{2}+x_{3} y_{3}\right)+i\left(x_{0} y_{1}+x_{1} y_{0}+x_{2} y_{3}+x_{3} y_{2}\right) \\
& +j\left(x_{0} y_{2}+x_{2} y_{0}+x_{1} y_{3}+x_{3} y_{1}\right)+k\left(x_{0} y_{3}+x_{3} y_{0}-x_{1} y_{2}-x_{2} y_{1}\right) .
\end{aligned}
$$


From properties of the operators of the sum and product of commutative-quaternions, for $p \in B Q$, we let the conjugate element $p^{*}$ such that $p^{*}=x_{0}-i x_{1}-j x_{2}+k x_{3}$. Then we have

$$
M(p):=p p^{*}=\left(x_{0}+k x_{3}\right)^{2}+\left(x_{1}+k x_{3}\right)^{2}
$$

and we set the element which appears $M(p)$ to a real number, denoted by $\overline{M(p)}$,

$$
\overline{M(p)}:=\left(x_{0}-k x_{3}\right)^{2}+\left(x_{1}-k x_{3}\right)^{2} .
$$

So, we give an inverse element $p^{-1}=\lambda_{0}+i \lambda_{1}+j \lambda_{2}+k \lambda_{3}$ such that $p p^{-1}=1$, where

$$
\begin{array}{ll}
\lambda_{0}=\frac{x_{0}\left(x_{0}^{2}+x_{1}^{2}+x_{2}^{2}-x_{3}^{2}\right)-2 x_{1} x_{2} x_{3}}{r^{4}}, & \lambda_{1}=\frac{x_{1}\left(-x_{0}^{2}-x_{1}^{2}+x_{2}^{2}-x_{3}^{2}\right)-2 x_{0} x_{2} x_{3}}{r^{4}}, \\
\lambda_{2}=\frac{x_{2}\left(-x_{0}^{2}+x_{1}^{2}-x_{2}^{2}-x_{3}^{2}\right)-2 x_{0} x_{1} x_{3}}{r^{4}}, & \lambda_{3}=\frac{x_{3}\left(-x_{0}^{2}+x_{1}^{2}+x_{2}^{2}+x_{3}^{2}\right)-2 x_{0} x_{1} x_{2}}{r^{4}},
\end{array}
$$

where a nonzero real number $r$ (in [12]) is

$$
\begin{aligned}
r^{4}=M(p) \overline{M(p)} & =\sum_{r=0}^{3} x_{r}^{4}+2\left(x_{0}^{2} x_{1}^{2}+x_{0}^{2} x_{2}^{2}-x_{0}^{2} x_{3}^{2}-x_{1}^{2} x_{2}^{2}+x_{1}^{2} x_{3}^{2}+x_{2}^{2} x_{3}^{2}\right)-8 x_{0} x_{1} x_{2} x_{3} \\
& \left.=\left\{\left(x_{0}^{2}-x_{3}^{2}\right)^{2}+\left(x_{1}^{2}-x_{2}^{2}\right)^{2}\right\}\left(x_{0}^{2}+x_{3}^{2}\right)^{2}+\left(x_{1}^{2}+x_{2}^{2}\right)^{2}\right\}-8 x_{0} x_{1} x_{2} x_{3} .
\end{aligned}
$$

If $x_{0}= \pm x_{3}, x_{1}= \pm x_{2}$, there is no inverse element in BQ.

\section{Hyperholomorphic functions of commutative-quaternionic variables}

Let $\Omega$ be an open subset in BQ. Let a commutative-quaternionic function $f$ of a commutativequaternionic variable $z$ be expressed by the real functions $f_{0}\left(x_{0}, x_{1}, x_{2}, x_{3}\right), f_{1}\left(x_{0}, x_{1}, x_{2}, x_{3}\right), f_{2}\left(x_{0}, x_{1}, x_{2}, x_{3}\right)$, and $f_{3}\left(x_{0}, x_{1}, x_{2}, x_{3}\right)$ of continuously differentiable real variables $x_{0}, x_{1}, x_{2}$, and $x_{3}$. We give relations of existences of the above limits between partial derivatives of the functions $f_{0}, f_{1}, f_{2}$, and $f_{3}$. From these relations, for $z_{0}=x_{0}^{0}+i x_{1}^{0}+j x_{2}^{0}+k x_{3}^{0}$, we obtain the derivative, denoted by $\frac{d f\left(z_{0}\right)}{d z}$ as follows.

Definition 3.1. Let a function $f: \Omega \rightarrow$ BQ be defined by

$$
f(z)=f_{0}\left(x_{0}, x_{1}, x_{2}, x_{3}\right)+i f_{1}\left(x_{0}, x_{1}, x_{2}, x_{3}\right)+j f_{2}\left(x_{0}, x_{1}, x_{2}, x_{3}\right)+k f_{3}\left(x_{0}, x_{1}, x_{2}, x_{3}\right),
$$

then the function $f$ is said to be hyperholomorphic on $\Omega$ if the following limit

$$
\frac{\mathrm{d} f\left(z_{0}\right)}{\mathrm{d} z}:=\lim _{z \rightarrow z_{0}}\left(z-z_{0}\right)^{-1}\left\{f(z)-f\left(z_{0}\right)\right\}=\lim _{\triangle z \rightarrow 0}(\Delta z)^{-1}\left(\frac{\partial f}{\partial x_{0}} \Delta x_{0}+\frac{\partial f}{\partial x_{1}} \Delta x_{1}+\frac{\partial f}{\partial x_{2}} \Delta x_{2}+\frac{\partial f}{\partial x_{3}} \Delta x_{3}\right),
$$

where

$$
\triangle z=z-z_{0}=\triangle x_{0}+i \triangle x_{1}+j \triangle x_{2}+k \triangle x_{3},
$$

with $\triangle x_{r}=x_{r}-x_{r}^{0}(r=0,1,2,3)$ and except for $x_{0}= \pm x_{3}, x_{1}= \pm x_{2}$, exists in BQ.

By the above definition of the derivative, we have the following equations:

$$
\left\{\begin{array}{l}
\frac{\partial f_{0}}{\partial x_{0}}=\frac{\partial f_{1}}{\partial x_{1}}=\frac{\partial f_{2}}{\partial x_{2}}=\frac{\partial f_{3}}{\partial x_{3}} \\
\frac{\partial f_{1}}{\partial x_{0}}=-\frac{\partial f_{0}}{\partial x_{1}}=-\frac{\partial f_{3}}{\partial x_{2}}=\frac{\partial f_{2}}{\partial x_{3}} \\
\frac{\partial f_{2}}{\partial x_{0}}=-\frac{\partial f_{3}}{\partial x_{1}}=-\frac{\partial f_{0}}{\partial x_{2}}=\frac{\partial f_{1}}{\partial x_{3}} \\
\frac{\partial f_{3}}{\partial x_{0}}=\frac{\partial f_{2}}{\partial x_{1}}=\frac{\partial f_{1}}{\partial x_{2}}=\frac{\partial f_{0}}{\partial x_{3}}
\end{array}\right.
$$


The above system is called the corresponding Cauchy-Riemann system for components of a commutativequaternionic function. From properties of components $f_{r}(r=0,1,2,3)$, we consider harmonic functions and solutions of the wave equation with respect to commutative-quaternionic variables.

Theorem 3.2. Let $\Omega$ be an open subset in $\mathrm{BQ}$ and a function $\mathrm{f}$ be hyperholomorphic in $\mathrm{BQ}$. Then each component of $\mathrm{f}$ is harmonic with respect to the pairs of variables $\left(x_{\mathrm{t}} ; \mathrm{x}_{\mathrm{r}}\right)(\mathrm{t}=0,3$ and $\mathrm{r}=1,2)$. Also, each component of $\mathrm{f}$ is a solution of the wave equation with respect to the pairs of variables $\left(\mathrm{x}_{0} ; \mathrm{x}_{3}\right)$ and $\left(\mathrm{x}_{1} ; \mathrm{x}_{2}\right)$.

Proof. Suppose the function $f$ is hyperholomorphic. Then we have the corresponding Cauchy-Riemann system (3.1) for components of a commutative-quaternionic function. From (3.1), we get

$$
\frac{\partial f_{0}}{\partial x_{0}}=\frac{\partial f_{1}}{\partial x_{1}}, \quad \frac{\partial f_{1}}{\partial x_{0}}=-\frac{\partial f_{0}}{\partial x_{1}} .
$$

We multiply by $\frac{\partial}{\partial x_{0}}$ and $\frac{\partial}{\partial x_{1}}$, respectively. Then we get

$$
\frac{\partial^{2} f_{0}}{\partial x_{0}^{2}}=\frac{\partial^{2} f_{1}}{\partial x_{0} \partial x_{1}}, \quad-\frac{\partial^{2} f_{1}}{\partial x_{1} \partial x_{0}}=\frac{\partial^{2} f_{0}}{\partial x_{1}^{2}} .
$$

Hence, we have

$$
\frac{\partial^{2} f_{0}}{\partial x_{0}^{2}}+\frac{\partial^{2} f_{0}}{\partial x_{1}^{2}}=0
$$

Also, we multiply

$$
\frac{\partial f_{0}}{\partial x_{2}}=\frac{\partial f_{2}}{\partial x_{0}} \text { and } \frac{\partial f_{2}}{\partial x_{2}}=-\frac{\partial f_{0}}{\partial x_{0}}
$$

by $\frac{\partial}{\partial x_{2}}$ and $\frac{\partial}{\partial x_{0}}$, respectively. Then,

$$
\frac{\partial^{2} f_{0}}{\partial x_{2}^{2}}=\frac{\partial^{2} f_{2}}{\partial x_{2} \partial x_{0}}, \quad \frac{\partial^{2} f_{2}}{\partial x_{0} \partial x_{2}}=-\frac{\partial f_{0}^{2}}{\partial x_{0}^{2}} .
$$

Hence, we have

$$
\frac{\partial^{2} f_{0}}{\partial x_{0}^{2}}+\frac{\partial^{2} f_{0}}{\partial x_{2}^{2}}=0
$$

For the following equations

$$
-\frac{\partial f_{0}}{\partial x_{1}}=\frac{\partial f_{2}}{\partial x_{3}} \text { and } \frac{\partial f_{2}}{\partial x_{1}}=\frac{\partial f_{0}}{\partial x_{3}}
$$

we multiply by $\frac{\partial}{\partial x_{1}}$ and $\frac{\partial}{\partial x_{3}}$, respectively. Then we have

$$
-\frac{\partial^{2} f_{0}}{\partial x_{1}^{2}}=\frac{\partial^{2} f_{2}}{\partial x_{1} \partial x_{3}}, \quad \frac{\partial^{2} f_{2}}{\partial x_{3} \partial x_{1}}=\frac{\partial f_{0}^{2}}{\partial x_{3}^{2}}
$$

and we obtain

$$
\frac{\partial^{2} f_{0}}{\partial x_{1}^{2}}+\frac{\partial^{2} f_{0}}{\partial x_{3}^{2}}=0
$$

Also, by $\frac{\partial}{\partial x_{2}}$ and $\frac{\partial}{\partial x_{3}}$, respectively, the equations

$$
-\frac{\partial f_{0}}{\partial x_{2}}=\frac{\partial f_{1}}{\partial x_{3}}, \quad \frac{\partial f_{1}}{\partial x_{2}}=\frac{\partial f_{0}}{\partial x_{3}}
$$

satisfy the following equation

$$
\frac{\partial^{2} f_{0}}{\partial x_{2}^{2}}+\frac{\partial^{2} f_{0}}{\partial x_{3}^{2}}=0
$$


For $f_{1}, f_{2}$, and $f_{3}$, by using the similar calculating with the above processes, we obtain for the pairs of variables $\left(x_{t} ; x_{r}\right)(t=0,3$ and $r=1,2)$, each components of $f$ is harmonic on $\Omega$.

Moreover, we multiply

$$
\frac{\partial f_{0}}{\partial x_{0}}=\frac{\partial f_{3}}{\partial x_{3}} \text { and } \frac{\partial f_{3}}{\partial x_{0}}=\frac{\partial f_{0}}{\partial x_{3}},
$$

by $\frac{\partial}{\partial x_{0}}$ and $\frac{\partial}{\partial x_{3}}$, respectively. Then we get

$$
\frac{\partial^{2} f_{0}}{\partial x_{0}^{2}}=\frac{\partial^{2} f_{3}}{\partial x_{0} \partial x_{3}} \text { and }-\frac{\partial^{2} f_{3}}{\partial x_{3} \partial x_{0}}=\frac{\partial^{2} f_{0}}{\partial x_{3}^{2}}
$$

Hence, we have

Also, we multiply

$$
\frac{\partial^{2} f_{0}}{\partial x_{0}^{2}}-\frac{\partial^{2} f_{0}}{\partial x_{3}^{2}}=0
$$

$$
-\frac{\partial f_{0}}{\partial x_{1}}=-\frac{\partial f_{3}}{\partial x_{2}} \text { and }-\frac{\partial f_{0}}{\partial x_{2}}=-\frac{\partial f_{3}}{\partial x_{1}}
$$

by $\frac{\partial}{\partial x_{1}}$ and $\frac{\partial}{\partial x_{2}}$, respectively. Then we have

$$
\frac{\partial^{2} f_{0}}{\partial x_{1}^{2}}-\frac{\partial^{2} f_{0}}{\partial x_{2}^{2}}=0
$$

By similar processing for the components $f_{1}, f_{2}$, and $f_{3}$, we also have the similar results. Therefore, for the pairs $\left(x_{0} ; x_{3}\right)$ and $\left(x_{1} ; x_{2}\right)$, each components of $f$ is a solution of the wave equation in $B Q$.

Let an orientation in the four dimensional space $\mathbb{R}^{4}$ be expressed with respect to the rotated system (in [12]) of

$$
\alpha=\frac{x_{0}+x_{3}}{\sqrt{2}}, \beta=\frac{x_{1}+x_{2}}{\sqrt{2}}, \gamma=\frac{x_{0}-x_{3}}{\sqrt{2}}, \delta=\frac{x_{1}-x_{2}}{\sqrt{2}} .
$$

The Figure 1 is said to be a hyperplane in BQ.

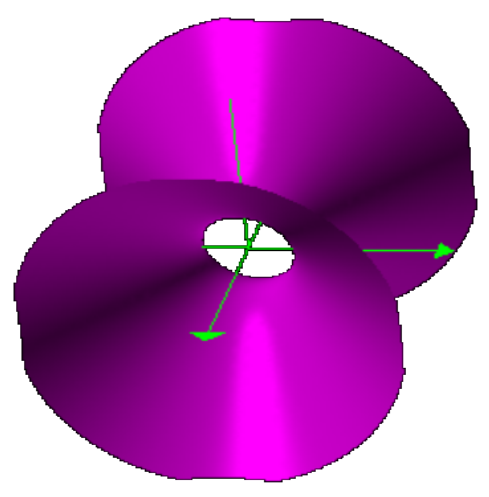

Figure 1: Hyperplane.

Theorem 3.3. Let $\Gamma$ be a surface consisting of a simple closed curve $\bar{\gamma}$ except for singularities of $\mathrm{f}$. If an integral of a commutative-quaternionic function $\mathrm{f}$ between two arbitrary points along a contour except for singularities is independent of the contour, then the integral of the hyperholomorphic function $\mathrm{f}$ along the curve $\bar{\gamma}$ is zero, that is,

$$
\oint_{\bar{\gamma}} f(z) d z=0
$$

where $d z=d x_{0}+i d x_{1}+j d x_{2}+k d x_{3}$. 
Proof. Consider the integration along the curve $\bar{\gamma}$ and the pole $z_{0}$, and their projections $\bar{\gamma}^{(\alpha \gamma)}, \bar{\gamma}^{(\alpha \delta)}$ and $z_{0}^{(\alpha \gamma)}, z_{0}^{(\alpha \delta)}$ on the planes $\alpha \gamma$ and $\alpha \delta$, respectively, as Figure 2.

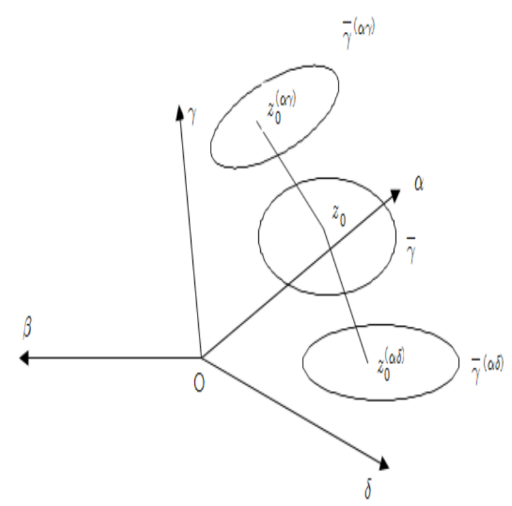

Figure 2: Projections on the planes.

Since either $z=z_{0}$ or all points of the two-dimensional hyperplanes being parallel to regions around $z_{0}$ as planes pass through $z_{0}$ and contain each $z_{0}, z_{0}^{(\alpha \gamma)}, z_{0}^{(\alpha \delta)}$ in Figure 2, by using the explicit form of the integral in the equation (3.2), we have

$$
\begin{aligned}
\oint_{\bar{\gamma}} f(z) d z= & \oint_{\bar{\gamma}}\left\{\left(f_{0} d x_{0}-f_{1} d x_{1}-f_{2} d x_{2}+f_{3} d x_{3}\right)+i\left(f_{1} d x_{0}+f_{0} d x_{1}+f_{3} d x_{2}+f_{2} d x_{3}\right)\right. \\
& \left.+j\left(f_{2} d x_{0}+f_{3} d x_{1}+f_{0} d x_{2}+f_{1} d x_{3}\right)+k\left(f_{3} d x_{0}-f_{2} d x_{1}-f_{1} d x_{2}+f_{0} d x_{3}\right)\right\} \\
= & \iint_{\Gamma}\left\{\left(\frac{\partial f_{0}}{\partial x_{1}}+\frac{\partial f_{1}}{\partial x_{0}}\right) d x_{1} d x_{0}+\left(\frac{\partial f_{0}}{\partial x_{2}}+\frac{\partial f_{2}}{\partial x_{0}}\right) d x_{2} d x_{0}\right. \\
& \left.+\left(\frac{\partial f_{0}}{\partial x_{3}}-\frac{\partial f_{3}}{\partial x_{0}}\right) d x_{3} d x_{0}+\left(\frac{\partial f_{1}}{\partial x_{2}}-\frac{\partial f_{2}}{\partial x_{1}}\right) d x_{2} d x_{1}+\left(\frac{\partial f_{1}}{\partial x_{3}}+\frac{\partial f_{3}}{\partial x_{1}}\right) d x_{3} d x_{1}+\left(\frac{\partial f_{2}}{\partial x_{3}}+\frac{\partial f_{3}}{\partial x_{2}}\right) d x_{3} d x_{2}\right\} .
\end{aligned}
$$

If each component of the function $f$ contain only the non-singularities on the surface $\Gamma$ with the curve $\bar{\gamma}$, by Stokes' theorem in the integral over the surface $\Gamma$, then we have

$$
\frac{\partial f_{0}}{\partial x_{1}}+\frac{\partial f_{1}}{\partial x_{0}}=\frac{\partial f_{0}}{\partial x_{2}}+\frac{\partial f_{2}}{\partial x_{0}}=0, \quad \frac{\partial f_{0}}{\partial x_{3}}-\frac{\partial f_{3}}{\partial x_{0}}=\frac{\partial f_{1}}{\partial x_{2}}-\frac{\partial f_{2}}{\partial x_{1}}=0,
$$

and

$$
\frac{\partial f_{1}}{\partial x_{3}}+\frac{\partial f_{3}}{\partial x_{1}}=\frac{\partial f_{2}}{\partial x_{3}}+\frac{\partial f_{3}}{\partial x_{2}}=0 .
$$

From the corresponding Cauchy-Riemann system (3.1), these are equal to zero. Therefore, we obtain

$$
\oint_{\bar{\gamma}} f(z) d z=0
$$

Example 3.4. For a function $z^{\mathfrak{n}}$ in $B Q$, since we have

$$
\int_{\bar{\gamma}} z^{\mathrm{n}} \mathrm{d} z=\frac{z^{\mathrm{n}+1}}{\mathrm{n}+1}
$$

and the function $z^{n+1}$ which contains the function for each integer $n$, the integral of the function $\frac{1}{z^{n}}$ on a simple closed curve $\bar{\gamma}$ is equal to zero for a positive or negative integer $n(n \neq-1)$, that is,

$$
\oint_{\bar{\gamma}} z^{\mathrm{n}} \mathrm{d} z=0
$$


Let $\mathrm{f}: \mathrm{U} \rightarrow \mathrm{BQ}$ be a function defined by $\mathrm{f}\left(z_{1}, z_{2}\right)=\varphi_{1}\left(z_{1}, z_{2}\right)+\mathrm{j} \varphi_{2}\left(z_{1}, z_{2}\right)$, where $\mathrm{U}$ is a bounded open set of $\mathbb{C}^{2}$ and

$$
\varphi_{1}\left(z_{1}, z_{2}\right)=f_{0}\left(x_{0}, x_{1}, x_{2}, x_{3}\right)+i f_{1}\left(x_{0}, x_{1}, x_{2}, x_{3}\right), \quad \varphi_{2}\left(z_{1}, z_{2}\right)=f_{2}\left(x_{0}, x_{1}, x_{2}, x_{3}\right)+i f_{3}\left(x_{0}, x_{1}, x_{2}, x_{3}\right)
$$

are complex analytic functions of $z_{1}=x_{0}+i x_{1}$ and $z_{2}=x_{2}+i x_{3}$ with real-valued functions $f_{r}(r=0,1,2,3)$. Consider the following differential operators:

$$
\mathrm{D}_{z}^{*}:=\frac{\partial}{\partial \overline{z_{1}}}+j \frac{\partial}{\partial \overline{z_{2}}}
$$

where

$$
\frac{\partial}{\partial \overline{z_{1}}}=\frac{\partial}{\partial x_{0}}+i \frac{\partial}{\partial x_{1}} \quad \text { and } \quad \frac{\partial}{\partial \overline{z_{2}}}=\frac{\partial}{\partial x_{2}}+i \frac{\partial}{\partial x_{3}} .
$$

Then the equation $D_{z}^{*} f=0$ satisfies the corresponding Cauchy-Riemann system (3.1). Indeed, $D_{z}^{*} f=0$ satisfies

$$
\frac{\partial \varphi_{1}}{\partial \overline{z_{1}}}=\frac{\partial \varphi_{2}}{\partial \overline{z_{2}}} \quad \text { and } \quad \frac{\partial \varphi_{1}}{\partial \overline{z_{2}}}=-\frac{\partial \varphi_{2}}{\partial \overline{z_{1}}} .
$$

Theorem 3.5. Let $\mathrm{U}$ be a bounded open set of $\mathbb{C}^{2}$. Let $\mathrm{f}: \mathrm{U} \rightarrow \mathrm{BQ}$ be a function defined by $\mathrm{f}\left(z_{1}, z_{2}\right)=$ $\varphi_{1}\left(z_{1}, z_{2}\right)+j \varphi_{2}\left(z_{1}, z_{2}\right)$. Then we have

$$
\int_{\mathrm{U}} \mathrm{f}\left(z_{1}, z_{2}\right)\left(\mathrm{d} \zeta_{1}+j \mathrm{~d} \zeta_{2}\right)=\iint_{\overline{\mathrm{U}}} \mathrm{D}_{\zeta}^{*} \mathrm{f}\left(\zeta_{1}, \zeta_{2}\right) j \mathrm{~d} \zeta_{1} \wedge \mathrm{d} \zeta_{2}
$$

where $\mathrm{D}_{\zeta}^{*}:=\frac{\partial}{\partial \bar{\zeta}_{1}}+\mathrm{j} \frac{\partial}{\partial \bar{\zeta}_{2}}, \mathrm{~d} \zeta_{1}=\mathrm{d} x_{0}+i \mathrm{~d} x_{1}, \mathrm{~d} \zeta_{2}=\mathrm{d} x_{2}+i \mathrm{~d} x_{3}$, and

$$
d \zeta_{1} \wedge d \zeta_{2}=d x_{0} \wedge d x_{2}-d x_{1} \wedge d x_{3}+i\left(d x_{0} \wedge d x_{3}+d x_{1} \wedge d x_{2}\right)
$$

Proof. We let $k=f_{0} d x_{0}-f_{1} d x_{1}-f_{2} d x_{2}-f_{3} d x_{3}$. Then we have

$$
\begin{aligned}
\mathrm{d} \kappa= & \left(\frac{\partial \mathrm{f}_{0}}{\partial x_{2}}+\frac{\partial \mathrm{f}_{2}}{\partial x_{0}}\right) d x_{2} \wedge d x_{0}+\left(\frac{\partial f_{0}}{\partial x_{3}}-\frac{\partial f_{3}}{\partial x_{0}}\right) d x_{3} \wedge d x_{0} \\
& +\left(\frac{\partial f_{2}}{\partial x_{1}}-\frac{\partial f_{1}}{\partial x_{2}}\right) d x_{2} \wedge d x_{1}-\left(\frac{\partial f_{3}}{\partial x_{1}}+\frac{\partial f_{1}}{\partial x_{3}}\right) d x_{3} \wedge d x_{1}-\left(\frac{\partial f_{3}}{\partial x_{2}}+\frac{\partial f_{2}}{\partial x_{3}}\right) d x_{3} \wedge d x_{2}+\left(\frac{\partial f_{0}}{\partial x_{1}}+\frac{\partial f_{1}}{\partial x_{0}}\right) d x_{1} \wedge d x_{0} .
\end{aligned}
$$

From the equations (3.3), we have

$$
\begin{aligned}
d \kappa= & \left(\frac{\partial f_{0}}{\partial x_{2}}+\frac{\partial f_{2}}{\partial x_{0}}\right)\left(d x_{2} \wedge d x_{0}-d x_{3} \wedge d x_{1}\right)+\left(\frac{\partial f_{0}}{\partial x_{3}}-\frac{\partial f_{3}}{\partial x_{0}}\right)\left(d x_{3} \wedge d x_{0}+d x_{2} \wedge d x_{1}\right) \\
& -\left(\frac{\partial f_{3}}{\partial x_{2}}+\frac{\partial f_{2}}{\partial x_{3}}\right) d x_{3} \wedge d x_{2}+\left(\frac{\partial f_{0}}{\partial x_{1}}+\frac{\partial f_{1}}{\partial x_{0}}\right) d x_{1} \wedge d x_{0} .
\end{aligned}
$$

Since $f$ satisfies equations (3.1), by using the result of $d \kappa$, we obtain

$$
\begin{aligned}
d \kappa= & -\left\{\left(\frac{\partial f_{2}}{\partial x_{0}}+\frac{\partial f_{0}}{\partial x_{2}}\right)+i\left(\frac{\partial f_{3}}{\partial x_{0}}+\frac{\partial f_{1}}{\partial x_{2}}\right)-j\left(\frac{\partial f_{0}}{\partial x_{0}}-\frac{\partial f_{2}}{\partial x_{2}}\right)-k\left(\frac{\partial f_{1}}{\partial x_{0}}-\frac{\partial f_{3}}{\partial x_{2}}\right)\right\} \\
& \cdot\left\{d x_{0} \wedge d x_{2}-d x_{1} \wedge d x_{3}+i\left(d x_{0} \wedge d x_{3}+d x_{1} \wedge d x_{2}\right)\right\} \\
= & \left\{\left(\frac{\partial \varphi_{1}}{\partial \overline{\zeta_{1}}}-\frac{\partial \varphi_{2}}{\partial \bar{\zeta}_{2}}\right) j-\left(\frac{\partial \varphi_{2}}{\partial \overline{\zeta_{1}}}+\frac{\partial \varphi_{1}}{\partial \overline{\zeta_{2}}}\right)\right\}\left(d \zeta_{1} \wedge d \zeta_{2}\right) \\
= & D \zeta f j d \zeta_{1} \wedge d \zeta_{2} .
\end{aligned}
$$

Therefore, by calculating and relating the above equations, we obtain equation (3.4). 


\section{Acknowledgment}

This work was supported by the Dongguk University Research Fund of 2017.

\section{References}

[1] P. Fjelstad, S. G. Gal, n-dimensional hyperbolic complex numbers, Adv. Appl. Clifford Algebras, 8 (1998), 47-68. 1

[2] R. Fueter, Analytische funktionen einer quaternionenvariablen, Comment. Math. Helv., 4 (1932), 9-20. 1

[3] J. D. Grant, I. A. B. Strachan, Hypercomplex Integrable Systems, Nonlinearity, 12 (1999), 1247-1261. 1

[4] W. R. Hamilton, On quaternions; or on a new system of imaginaries in algebra, Lond. Edin. Dublin Phil. Mag. J. Sci., 25 (1844), 58-60. 1

[5] J. Kajiwara, Z. Li, K. H. Shon, Function spaces in complex and Clifford analysis, Hue University, Vietnam, (2006). 1

[6] D. Kaledin, Integrability of the twistor space for a hypercomplex manifold, Selecta Math., 4 (1998), 271-278. 1

[7] J. E. Kim, S. J. Lim, K. H. Shon, Regularity of functions on the reduced quaternion field in Clifford analysis, Abstr. Appl. Anal., 2014 (2014), 8 pages. 1

[8] J. E. Kim, K. H. Shon, The Regularity of functions on Dual split quaternions in Clifford analysis, Abstr. Appl. Anal., 2014 (2014), 8 pages. 1

[9] J. E. Kim, K. H. Shon, Polar coordinate expression of hyperholomorphic functions on split quaternions in Clifford analysis, Adv. Appl. Clifford Algebr., 25 (2015), 915-924. 1

[10] V. V. Kravchenkov, Applied Quaternion Analysis, Helderman, Berlin, (2003). 1

[11] K. Nôno, Hyperholomorphic functions of a quaternion variable, Bull. Fukuoka Univ. Ed. III, 32 (1982), 21-37. 1

[12] S. Olariu, Complex numbers in $n$ dimensions, North-Holland Publishing Co., Amsterdam, (2002). 1, 2, 3

[13] K. Scheicher, R. F. Tichy, K. W. Tomantschger, Elementary Inequalities in Hypercomplex Numbers, Anz. Österreich. Akad. Wiss. Math.-Natur. Kl., 134 (1997), 3-10. 1

[14] A. Sudbery, Quaternionic Analysis, Math. Proc. Cambridge Philos. Soc., 85 (1979), 199-224. 1 